\title{
Neoplasias em DII: implicações terapêuticas
}

Fernando Magro

Inflammatory bowel disease (IBD)-related cancers are those attributable to chronic tissue inflammation and/or to drug-induced immunosuppression. Extra-intestinal cancers belong to the second category. This review focus on lymphoproliferative disorders (LD), skin cancers and uterine cervix abnormalities.

\section{Lymphoproliferative disorders}

Lymphoproliferative disorders (LD) in inflammatory bowel disease (IBD) have become a major concern for both patients and physicians. The role of immunosuppressive therapy, as well as the role of local and systemic inflammation, is interlinked, and thus difficult to distinguish. The incidence of LD has increased markedly worldwide since the 1970s and particularly in western countries. The current lifelong risk for a male reached approximately $2 \%$. Although case series and hospital-based studies suggested an increased risk of LD in IBD patients, population-based studies showed no increase or very little increase risk of $L D$ in $I B D^{1-4}$. Immunosuppression is one of the well established risk factor for LD. In the post-transplant setting, EBV viral load is a good marker of the risk of LD ${ }^{5}$ but this strategy has not been evaluated in IBD. Genetic factors are involved in LD because familial clusters have been described. In addition, EBV and other specific infectious agents have also been involved, such as human herpes virus 8 
(HHV-8) and human T lymphotropic virus 1 (HTLV-1). In autoimmune diseases, such as lupus erythematosus, Hashimoto thyroiditis and Sjögren's syndrome, the risk of LD is increased 3-to 18-fold and seems to correlate with disease severity. Moreover, LD are often located in the organ in which occurs the auto-immune reaction ${ }^{6}$. In IBD has been shown an excess risk of primary intestinal $\mathrm{LD}^{7}$ and in CESAME study the duration of the disease is an independent risk factor for $L^{1}$.

Thiopurines have been particularly implicated in IBD-associated LD risk. Thiopurines might have specific pro-carcinogenic effects by promoting clonal expansion of rare mismatch repair (MMR)-defective myeloid cells. Meta-analysis of cohort studies and nationwide studies has shown that patients with IBD exposed to thiopurines have a three- to fivefold increased risk of developing LD $^{1,8}$. Several cases of LD have been reported in methotrexate-treated patients with RA, but a French nationwide study showed that the risk of non-Hodgkin lymphoma was not significantly increased in patients receiving methotrexate $^{9}$. Although no similar studies are available (or will be available in the near future) in IBD, the risk of LD associated with methotrexate seems to be low in $\mathrm{IBD}^{10}$. The risk of $L D$ in patients under anti-TNF is difficult to estimate, however analysis of the five-year follow-up of the Crohn's Therapy, Resource, Evaluation, and Assessment Tool (TREAT) registry, showed a similar overall incidence of LD in infliximab-treated patients ( 0.05 per 100 patient-years) and those who received other treatments only (0.06 per 100 patient-years $)^{11}$.

Based on the epidemiology of HSTCL in IBD, it seems reasonable to consider avoiding association of anti-TNF therapy and thiopurine treatment beyond two years in young ( $<35$ years) men. Regarding the risk of post-mononucleosis lymphoproliferation, thiopurine treatment should be avoided in young ( $<35$ years) men seronegative for EBV. In this line, systematic EBV serology before initiating immunosuppressants should be discussed ${ }^{11}$.

\section{Skin Cancer}

Non-melanoma skin cancer (NMSC) is the most common human cancer. Melanomas are much less frequent than NMSC, but the incidence of melanomas continues to increase worldwide. Previous experience from transplant recipients indicates that exposure to thiopurines 
is associated with an increased risk of NMSC. A recent meta-analysis showed that anti-TNF treatment increases the risk of NMSC, and possibly that of melanoma among patients with rheumatoid arthritis ${ }^{12}$. In IBD the ongoing and past exposure to thiopurines significantly increases the risk of NMSC in patients with IBD. The risk of melanoma in patients who receive thiopurines for IBD is not increased whereas this risk is increased by the use of biologics ${ }^{13}$. Long et al., found an incidence rate ratio of NMSC of 1.64 (95\% confidence interval [Cl] = 1.51-1.78) among IBD patients compared to controls ${ }^{14}$. In the CESAME cohort study, an excess of NMSC was observed in the overall IBD population (SIR, 2.89; $95 \% \mathrm{Cl}=1.98-4.08)^{15}$ while the risk of melanoma among the overall IBD population was similar to that observed in the general population $(\mathrm{SIR}, 0.64 ; 95 \% \mathrm{Cl}=0.17-1.63)^{16}$. The increased risk of NMSC in IBD patients who receive thiopurines is now well-documented, with HR of $5.9(95 \% \mathrm{Cl}=2.1-16.4 ; \mathrm{p}=.0006)$ for ongoing treatment and $3.9(95 \%$ $\mathrm{Cl}=1.3-12.1 ; \mathrm{p}=.02$ ) for past exposure in the CESAME cohort study. Long et al. found that thiopurine use ( $\leq 90$ days) and persistent thiopurine use (>365 days) were associated with NMSC, with adjusted odds ratio [OR] of $3.56(95 \% \mathrm{Cl}=2.81-4.50)$ and $4.27(95 \% \mathrm{Cl}=3.08-$ 5.92), respectively ${ }^{14}$. The use of methotrexate or calcineurin inhibitors (tacrolimus or cyclosporine) was not significally associated with NMSC in IBD patients ${ }^{14}$. Combinations of immunosuppressants and biologics were associated with NMSC risk in CD patients, with an adjusted OR of 5.85 (95\% Cl = 3.2-10.8; $\mathrm{p}<0.001)$ and $6.75(95 \% \mathrm{Cl}=2.74-16.65$; $p<0.001$ ) for recent use ( $\leq 90$ days) and persistent use (> 365 days), respectively ${ }^{14}$. In the CESAME cohort study, the risk of melanoma was not increased in IBD patients previously treated with thiopurines (SIR, $0 ; 95 \% \mathrm{Cl}=0-3.11)^{16}$. In a recent study, the risk of melanoma was increased in IBD patients with biologics (OR, 1.88; 95\% Cl = 1.08-3.29) ${ }^{13}$. A regular dermatological screening and protection against UV radiation are recommended lifelong for all IBD patients receiving immunosuppressive therapy.

\section{Uterine cervix abnormalities}

Cervical cancer is the second most common cancer in women worldwide, and the incidence of cervical cancer is higher in developing countries than in developed countries that developed an effec- 
tive cervical screening program. The worldwide prevalence of human papillomavirus (HPV) infection in women is about 10\%. High-risk HPV is now considered to be the causal agent of almost all cases of invasive cervical cancer and cervical intra-epithelial neoplasia (CIN). HPV has been identified as the 'necessary cause' of cervical cancer. In other words, cancer does not develop in the absence of persistent HPV DNA ${ }^{17}$. HPV types 16, 18, 45 and 31 are the most ongogenic types, while HPV-6 and 11 cause benign warts and lesions that do not become malignant. Types 16 and 18 together are the causative agent of more than $50 \%$ of cervical pre-cancerous lesions (high-grade dysplasia), as well as more than $70 \%$ of all cases of cervical squamous cell carcinoma and adenocarcinoma, and 25\% of low-grade cervical lesions ${ }^{18}$. Several risk factors have been associated with the development of cervical cancer, namely prolonged combination oral contraceptive use, number of sexual partners, parity, early age at first intercourse (before the age of 20 years), tobacco smoking, coinfection with other sexually transmitted agents, concomitant infection with multiple HPV types, HPV viral load, low social class, and multiplicity of sexual partners. In IBD current smokers, women aged of less than 20 years at diagnosis, extensive disease, exposure to 10 or more prescriptions of oral contraceptives, and oral contraceptives were found as risk factors for cervical dysplasia. However, in most of these studies, important HPV risk factors were not evaluated, such as the number of sexual partners, sexual transmitted diseases and education level ${ }^{10}$. Previous studies have demonstrated an increased prevalence of atypical cervical smears in patients with systemic lupus erythematosus, in those exposed to cyclosphosphamide and infrcted with HIV ${ }^{10}$.

In IBD two population-based studies, one from North of California, and the other from Manitoba, were published. The former ${ }^{19}$, retrospective, showed an adjusted odds ratio (OR) of 1.45 for risk of cervical cancer in IBD patients. Odds ratios associated with drug use were 1.65 for 5-aminosalicylates, 2.79 for corticosteroids, and 3.45 for immunomodulators (all $\mathrm{P}<0.05$ ). The latter was a case-control study nested in a population-based cohort ${ }^{20}$. There was no association between cervical abnormalities and ulcerative colitis. The increase in risk in women with Crohn's disease was limited to those exposed 
to 10 or more prescriptions of oral contraceptives. The combined exposure to corticosteroids and immunosuppressants was associated with an increased risk of cervical abnormalities. There was no interaction between the effect of IBD and corticosteroids and/or immunosuppressants.

In a retrospective study from a tertiary care center in Scotland ${ }^{21}$, 362 women with IBD were compared with 1,644 controls from the community. There was no difference in the proportion of abnormal smears between women with IBD and matched controls. There was no association with exposure to any immunosuppressant specifically, even when limited to women who were on drug at the time of smear. No definite conclusion on the role of exposure to immunosuppressants on the risk of cervical cancer in IBD patients can be made at the moment. In addition, we have few data on sexual activity and frequency of cervical screening in the IBD population, with or without immunosuppressants. It was reported that only $20 \%$ of women with IBD report moderate to high sexual activity and it is known that sexual activity and the number of partners have an impact on the rate of cervical dysplasia. Immunosuppressants were found independent predictors of lower use of Pap testing in IBD. The prevalence of surveillance in the different studies was small and varied from 11 to $65 \%{ }^{10}$. Older age, lower socioeconomic status, lower intensity of healthcare utilization, Crohn's disease, and exposure to immunosuppressants were independent predictors of lower use of Pap testing. Gastroenterologists should strengthen recommendations of routine Pap smears in women with IBD.

Cytological screening at the population level every 3-5 years results in a substantial reduction (up-to-80\%) in the incidence of cervical cancer. Cervical cytology is the currently recommended standard test for cervix screening, which should start in the age range of 20-30 years. It is recommended to continue screening at 3- to 5-year intervals until the age of 60 or $65^{22}$. Stopping screening in older women is probably appropriate among women who have had three or more consecutive previous (recent) normal cytology results. Women under 30 years of age should not be screened for HPV, due to the high rate of viral clearance. Young women who are infected with HIV and/or are immunocompromised should obtain a Pap test twice in the first year after diagnosis of 
HIV infection and, if the results are normal, annually thereafter ${ }^{10}$. For immunocompromised males, ACIP (Advisory Committee on Immunization Practices) recommends routine vaccination as for all females, and vaccination through age 26 years for those who have not been vaccinated previously or who have not completed the 3-dose series. ACIP recommends routine vaccination of females aged 11-12 years. Vaccination is also recommended for females aged 13-26 years who have not been previously vaccinated or who have not completed the full series. Ideally, vaccine should be administered in sexually naïve individuals, before potential genital exposure to HPV. However, females who might have already been exposed to HPV should be vaccinated. Sexually active females who have not been infected with any of the HPV vaccine types would receive full benefit from vaccination. It seems that vaccination provides less benefit to females who have already been infected with one or more of the four vaccine HPV types. Pap testing and screening for HPV DNA or HPV antibody are not needed before vaccination at any age (http://www.cdc.gov/mmwr/preview/mmwrhtml/rr5602a1.htm)

\section{References:}

1. Beaugerie L, Brousse N, Bouvier AM, et al. Lymphoproliferative disorders in patients receiving thiopurines for inflammatory bowel disease: a prospective observational cohort study. Lancet 2009;374:1617-25.

2. Ekbom A, Helmick C, Zack M, et al. Extracolonic malignancies in inflammatory bowel disease. Cancer 1991;67:2015-9.

3. Lewis JD, Bilker WB, Brensinger C, et al. Inflammatory bowel disease is not associated with an increased risk of lymphoma. Gastroenterology 2001;121:1080-7.

4. Loftus EV, Jr., Tremaine WJ, Habermann TM, et al. Risk of lymphoma in inflammatory bowel disease. Am J Gastroenterol 2000;95:2308-12.

5. Stevens SJ, Verschuuren EA, Pronk I, et al. Frequent monitoring of Epstein-Barr virus DNA load in unfractionated whole blood is essential for early detection of posttransplant lymphoproliferative disease in high-risk patients. Blood 2001;97:1165-71.

6. Bosetti C, Levi F, Ferlay J, et al. Incidence and mortality from non-Hodgkin lymphoma in Europe: the end of an epidemic? Int J Cancer 2008;123:1917-23.

7. Sokol H, Beaugerie L, Maynadie M, et al. Excess primary intestinal lymphoproliferative disorders in patients with inflammatory bowel disease. Inflamm Bowel Dis 2012; Nov;18(11):2063-71.

8. Kandiel A, Fraser AG, Korelitz BI, et al. Increased risk of lymphoma among inflammatory bowel disease patients treated with azathioprine and 6-mercaptopurine. Gut 2005;54:1121-5.

9. Mariette X, Cazals-Hatem D, Warszawki J, et al. Lymphomas in rheumatoid arthritis patients treated with methotrexate: a 3-year prospective study in France. Blood 2002;99:3909-15.

10. Magro F, Peyrin-Biroulet L, Sokol H, Aldeger X, Costa A, Higgins, PD, Joyce JC, Katsanos KH, Lopez A, de Xaxars TM, Toader E, Beaugerie L. Extra-intestinal malignancies in inflammatory bowel disease: results of the 3rd ECCO Pathogenesis Scientific Workshop (III). J Crohns Colitis. 2014 Jan;8(1):31-44.

11. Lichtenstein GR, Feagan BG, Cohen RD, et al. Serious Infection and Mortality in Patients With Crohn's Disease: More Than 5 Years of Follow-Up in the TREAT Registry. Am J Gastroenterol 2012;107:1409-22. 
12. Mariette X, Matucci-Cerinic M, Pavelka K, et al. Malignancies associated with tumour necrosis factor inhibitors in registries and prospective observational studies: a systematic review and meta-analysis. Ann Rheum Dis 1895;70:1895-904.

13. Long MD, Martin CF, Pipkin CA, et al. Risk of melanoma and nonmelanoma skin cancer among patients with inflammatory bowel disease. Gastroenterology 2012;143:390-399

14. Long MD, Herfarth HH, Pipkin CA, et al. Increased risk for non-melanoma skin cancer in patients with inflammatory bowel disease. Clin Gastroenterol Hepatol 2010;8:268-74.

15. Peyrin-Biroulet L, Khosrotehrani K, Carrat F, et al. Increased risk for nonmelanoma skin cancers in patients who receive thiopurines for inflammatory bowel disease. Gastroenterology 2011;141:1621-28.

16. Peyrin-Biroulet L, Chevaux JB, Bouvier AM, et al. Risk of melanoma in patients who receive thiopurines for inflammatory bowel disease is not increased. Am J Gastroenterol 2012;107:1443-4.

17. Bosch FX, Lorincz A, Munoz N, et al. The causal relation between human papillomavirus and cervical cancer. Journal of Clinical Pathology 2002;55:244-265.

18. Galani E, Christodoulou C. Human papilloma viruses and cancer in the post-vaccine era. Clinical Microbiology and Infection 2009;15:977-981.

19. Hutfless S, Fireman B, Kane S, et al. Screening differences and risk of cervical cancer in inflammatory bowel disease. Alimentary Pharmacology \& Therapeutics 2008;28:598-605

20. Singh H, Demers AA, Nugent Z, et al. Risk of Cervical Abnormalities in Women With Inflammatory Bowel Disease: A Population-Based Nested Case-Control Study. Gastroenterology 2009;136:451-458.

21. Lees CW, Critchley J, Chee N, et al. Lack of Association Between Cervical Dysplasia and IBD: A Large Case-Control Study. Inflammatory Bowel Diseases 2009;15:1621-1629.

22. Arbyn M, Anttila A, Jordan J, et al. European Guidelines for Quality Assurance in Cervical Cancer Screening. Second Edition-Summary Document. Annals of Oncology 2010;21:448-458. 